\title{
USABILITY ENGINEERING IN PATIENTS FOLLOW-UP MEDICAL INFORMATION SYSTEMS
}

\author{
Miguel A. Valero, M. Teresa Arredondo, Marcos F. Sanz, Francisco del Pozo \\ Grupo de Bioingeniería y Telemedicina (GBT). Departamento de Tecnología Fotónica (TFO). \\ E.T.S.I. Telecomunicación. Universidad Politécnica de Madrid, España. \\ Ciudad Universitaria s/n. 28040 Madrid SPAIN \\ mavalero@teb.upm.es
}

\begin{abstract}
This paper tries to show that the key quality attribute to introduce information systems in health environments at present is the usability obtained with human-computer interaction techniques (HCI). A neonathology management system was selected to show the application of a usability engineering process. In order to achieve high usability levels, a specific methodology was followed and specialized medical users periodically took part in the design process. The methodology is essentially an effective mixture of $\mathrm{HCI}$ techniques with minimum cost. This system provides medical professionals with all the information required to check and follow up patients periodically. The results show that not only the users accepted the system in their work environment, but they spontaneously increased their participation in the design as they benefited from the system. Even, they proposed new system features and capabilities that allow to improve the health care.
\end{abstract}

Keywords

Usability, user requirements, user-oriented interface design.

\section{INTRODUCTION}

The field of HCI might play a key role in medical information systems. For diverse reasons, medical field has underutilized technology in support of its work. Thanks to significant developments in Information Technologies, this situation will probably get better in the following years.

An integrated work with medical fields requires $\mathrm{HCl}$ techniques which allow to study in depth clinical contexts. These studies supply basic information that guarantees wide acceptance from all users. This interface was designed starting from this learned knowledge. Later on, the continuous dialogue with the specialties improved the previous definition of the user requirements.

The main goal of this system is to ease the access to the necessary information to do and evaluate periodically followup checks to patients. This objective demands specific methods which guarantee an optimal usability level. The methodology followed to design the graphical user interface (UI) [1] has been developed in our group (GBT). Its application has required the constant collaboration of the neonathology department at the University Hospital San Carlos in Madrid, Spain.

\section{METHODS}

The methodology to design and develop the graphical UI combines, in a cost effective manner, the following activities: the modeling of requirements at a high level of abstraction, that obtains the context of use and a formal Task Model (TM) at the semantic level of user task (it includes the concept of semantic enabling tasks, as a refinement of enabling task concept [2], which considers technological limitations during the specification of the tasks supported by the system); the translation of the TM (semantic objects and task plan) into the system semantic domain by using metaphors of graphical objects at the semantic level of description [3]; the development of UI syntax and articulation using a real world or an abstract metaphor, without excluding artistic contributions; the evaluation of the TM, metaphors, syntax and articulation (using prototypes of the UI) through iterative cycles.

\section{RESULTS}

Next follows a description of how usability engineering was applied in an Follow-up Medical Information System.

\section{Product Description}

This product is a software tool that supplies useful information to carry out the follow-up care of neonathology patients. It was designed to be used in any environment related to a child growth follow-up (health and education areas). However, its use is generalizable to any setting which requires a strict follow-up of people or events.

\section{Context of Use Description}

A Context of Use Questionnaire allowed to specify all factors which affect the product usability. The product should be very easy to use since users' background in computers was limited. The description of tasks showed how and how often medical users do their work. The technical environment description added the obligation of using computers as single as the ones used in the hospital.

\section{Task and Enabling Tasks Analysis}

The most important problems found in the Tasks Analysis were: to find out what tasks should be decomposed and the limits of decomposition. The first question was solved by defining with users all the system independent tasks. These tasks defined the procedures followed by doctors at their daily work and are the only ones who were decomposed. This 
was a hard work since users' tasks definition was not clear, and some users already worked with computers. Besides, another difficulty appeared: the product offers new functions which are hard to do without the system. This fact required users to test the system in order to observe changes in the way they traditionally done tasks. The limits of decomposition stopped when further decomposition did not offer great help to develop the interface, or new resulting tasks implied system dependent tasks.

The semantic enabling tasks, that depend on the "hardware" and "software" specification, were minimized in order to ease to the utmost the apprenticeship of the system.

\section{Graphical specification and user interface development}

Independent tasks showed which ones should be present in the interface at the same time. This allows the doctor to interrupt his or her work if needed. All metaphors designed make use of the existing knowledge about objects which are familiar to users in real world. E.g., before the system design, doctors started to use small cards to collect data of explorations. However, the high number of cards for each patient made impossible the application of this method. Thanks to the system abilities, the metaphor of cards was developed because users accepted it as the best way to arrange high amounts of structured information. This metaphor may be used in any other interface which manages a lot of data. Experience showed that "direct manipulation" of cards in combination with the use of a card index (Figure 1) is a very easy way to manage information.

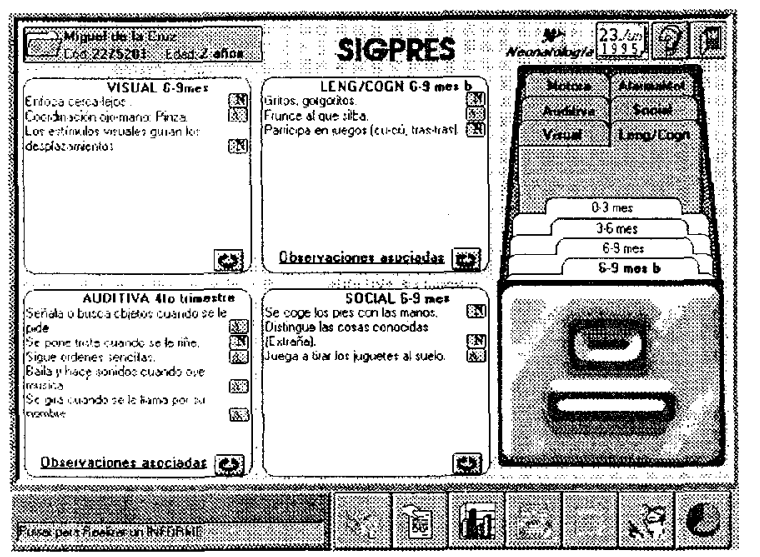

Figure 1. Card index, separators and cards with neuroevolutive explorations.

\section{Usability evaluation}

Usability was evaluated with objective and subjective methods. Six users with low experience with computers were filmed as they performed some tasks. The time needed to finish their tasks was lower than expected and it decreased with use. The time spent was strongly dependent of their ability with mouse and keyboard. Three groups of six people from the staff of the hospital evaluated satisfactorily the system through extensive subjective questionnaires. Cyclic evaluation during design process improved the system usability and users quickly familiarized themselves with the product.

\section{CONCLUSIONS}

The main conclusions extracted from this work are:

- The UI design methodology helps to increase substantially the system usability by focusing on the earliest design stages.

- High usability graphical interfaces development need from powerful information systems with high graphical possibilities that secures operation speed and graphical resolution to the interface.

- System usability has been well evaluated but requires more evaluation from a bigger number of specialized users from other environments. Professional users not only accepted the system, but involved themselves in the design process and even proposed new system features and capabilities that allow an improvement of their work in health care.

\section{ACKNOWLEDGMENTS}

Dr. J. Arizcun, head of the neonathology department at university hospital San Carlos in Madrid. Miguel Valles, research fellow in the GBT from ETSIT of Madrid, Spain.

\section{REFERENCES}

1. Sanz, Gómez. Task Model for Graphical User Interface Development. Technical Report gbt-hf-95-1, March 95, <http://www.teb.upm.es/TechReports_E.html >

2. Byerley, P., May, J., Whitefield, A., Denley, I. The Enabling States Approach: Designing Usable Telecommunications Services. IEEE Journal on Selected Areas in Communications, May, 1991.

3. Hammond, N.V., Allison, L.J. The travel metaphor as design principle and training aid for navigating around complex systems. People and Computers III. Cambridge University Press, 1987. 